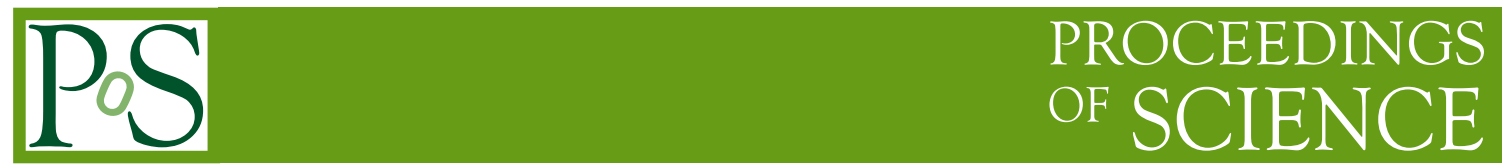

\title{
Low mass Higgs and Higgs properties at the LHC
}

\section{Junichi TANAKA ${ }^{* i}$}

International Center for Elementary Particle Physics (ICEPP), University of Tokyo

E-mail: Junichi.Tanaka@cern.ch

\begin{abstract}
The discovery of the Higgs boson is one of the most important studies at the LHC to understand the mechanism of the electroweak symmetry-breaking and the origin of mass for elementary particles. We present results of $H \rightarrow \tau \tau$ and $H \rightarrow \gamma \gamma$ analysis based on the latest simulation with $14 \mathrm{TeV}$ center of mass energy at the LHC. The mass region explored by these channels is around $110-150 \mathrm{GeV}$, that is preferred by the existing precision and direct measurements (LEP, Tevatron and so on). We also briefly discuss the result of coupling measurements.
\end{abstract}

The 2009 Europhysics Conference on High Energy Physics,

July 16 - 222009

Krakow, Poland

*Speaker.

${ }^{\dagger}$ On behalf of ATLAS and CMS collaboration 


\section{Introduction}

The discovery of the Higgs boson is one of the most important studies at the LHC to understand the mechanism of the electroweak symmetry-breaking and the origin of mass for elementary particles. This is a crucial goal of the LHC experiments ATLAS [1] and CMS [2]. A Standard Model Higgs is expected to be lighter than about $157 \mathrm{GeV}$ (95\% C.L., August 2009) with the precision measurements and is bound to be heavier than $114 \mathrm{GeV}$ by the direct search at LEP. Tevatron has recently excluded the region of $160-170 \mathrm{GeV}$. Once a Higgs-like particle is discovered, its properties, mass, width, spin, CP and couplings will be measured at the LHC. In this paper, we show results of $H \rightarrow \tau \tau$ and $H \rightarrow \gamma \gamma$ analyses ${ }^{1}$ based on the latest simulation with $14 \mathrm{TeV}$ center of mass energy and also the result expected on the coupling measurements.

\section{Higgs search}

\subsection{VBF $H \rightarrow \tau \tau$}

Higgs production via vector boson fusion (VBF) has a relatively large cross-section (a few pb) and provides a better background rejection thanks to the next two characteristics; 1) two out-going quarks have larger $p_{T}$ than QCD background because of the exchange of $\mathrm{W}$ and $\mathrm{Z}$ bosons. They will be observed in a forward region with high $p_{T}$. 2) since there is no color exchange in the central region, decay products from a Higgs will be observed in a large rapidity gap, where the activity of QCD jets is small. Tagging these forward jets and the veto of additional jets in the central region allows to suppress the backgrounds.

(a)

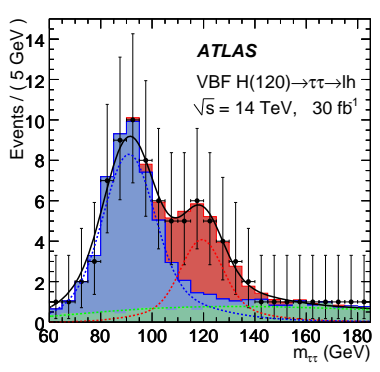

(b)

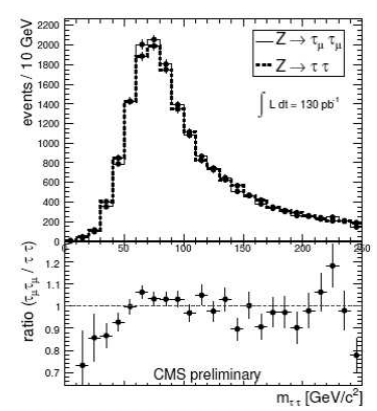

Figure 1: (a) the invariant mass of Higgs candidates of VBF $H \rightarrow \tau \tau \rightarrow l h$ analysis (red is signal, blue $Z \rightarrow \tau \tau$, green others $(t \bar{t})$ ) (b) the invariant mass of $Z \rightarrow \tau \tau$ from the embedding method (solid) and reference (dotted) and its ratio.

As shown in Figure 1(a) [3] ${ }^{2}$, ATLAS can reconstruct a Higgs mass using a collinear approximation method with $\not_{T}$. The improvement of $\mathbb{E}_{T}$ resolution is one of key issues since the Higgs mass resolution largely depends on it. Also the understanding of a long tail of Drell-Yan is important to extract signal events. This shape can be estimated from $Z \rightarrow \mu \mu$ data by replacing $\mu$

\footnotetext{
${ }^{1} H \rightarrow W W, H \rightarrow Z Z$ and combined discovery potential results at the LHC were presented by Cristina BOTTA.

${ }^{2}$ There are 3 different analyses based on a tau decay mode; $l h$ stands for leptonically and hadronically decaying taus, $l l$ for 2 leptonically and $h h$ for 2 hadronically.
} 
to $\tau$ (embedding method). Figure 1(b) shows that an estimated shape (solid) by this method can well describe $Z \rightarrow \tau \tau$ (dotted) [4].

Figures 2(a) and (b) show ATLAS and CMS expected signal significance, respectively [3, 4]. About 5 sigma significance can be achieved with $30 \mathrm{fb}^{-1}$ of data and the CMS figure shows that we can observe this channel $(l h)$ with $1 \mathrm{fb}^{-1}$ of data if its cross section is larger than the SM prediction by a factor of $\sim 9$. This could be improved further by adding $l l$ and also $h h$ channels.

(a)

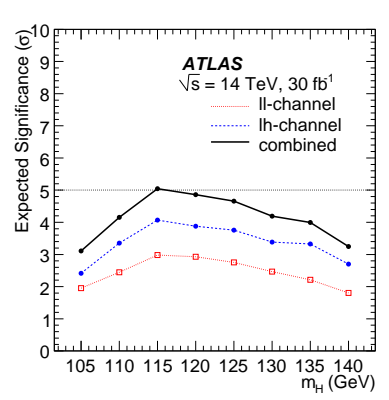

(b)

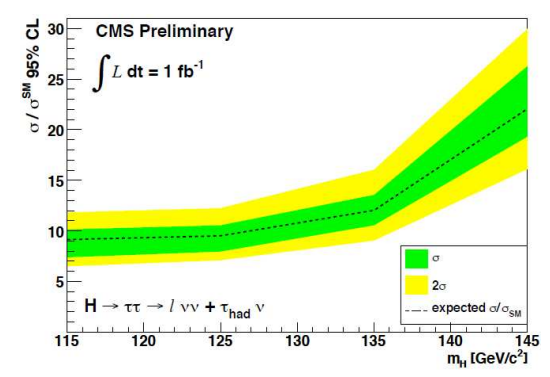

Figure 2: (a) the expected signal significance at ATLAS and (b) the 95\% CL limit on the SM prediction at CMS for VBF $H \rightarrow \tau \tau \rightarrow l h$.

\section{$2.2 H \rightarrow \gamma \gamma$}

The branching ratio of $H \rightarrow \gamma \gamma$ is very small $(\sim 0.2 \%)$ but a narrow mass peak is observed on a huge, smooth background as shown in Figure 3(a) [3] (ATLAS inclusive analysis). S/B is 0.027. There are two types of background; $\gamma \gamma+$ jets (irreducible) and $\gamma+$ jets and QCD with fake photons (reducible). The photon fake rate from jets need to be estimated from data.

Figure 3(b) shows an invariant mass distribution of $\gamma \gamma$ of signal and its shaded (yellow) histogram shows a contribution of $\gamma \gamma$ with at least one conversion. This fraction is about $60 \%$ of $H \rightarrow \gamma \gamma$. Due to a worse energy calibration to conversions, this mass distribution has a tail in the lower side. This could be improved by using a different energy calibration to conversions from uncovered photons. Materials in front of EM calorimeter will be understood by using conversions.

Additional jets can be required in order to improve S/B since the leading jet tends to be harder for the signal than for backgrounds or to enhance VBF contribution. Figure 4(a) show the expected performance for the 2 jets analysis in ATLAS. S/B is 0.082 (1jet) and 0.50 (2jets), which is improved compared to the inclusive analysis even if the number of signal events becomes small.

Figure 4(b) shows signal significance for 3 different mass points with several methods. From the counting method shown by closed and open circles, the signal significance of $\sim 2.6$ (only inclusive) and $\sim 3.3$ (combined) can be achieved with $10 \mathrm{fb}^{-1}$ of data at ATLAS [3]. CMS also has similar results and the details are in [5].

\section{Coupling measurement}

In the low mass region (110-150 GeV), since event rates of Higgs boson are measured in several channels, the coupling information can be extracted. The determination of relative couplings is possible in a model independent way. Figure 4(c) shows uncertainties of measurement of relative 
(a)

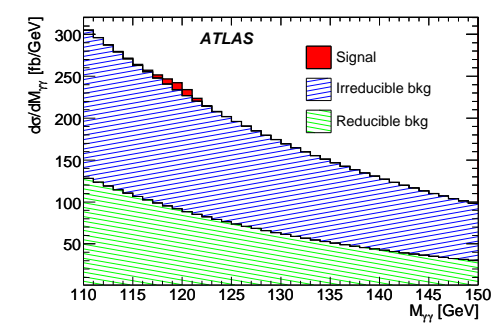

(b)

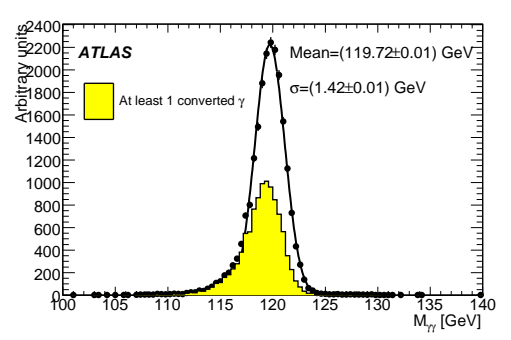

Figure 3: (a) the invariant mass of $\gamma \gamma$ of signal and background with the inclusive analysis and (b) of only signal, where a contribution of $\gamma \gamma$ with at least one conversion is shown by shaded (yellow).

(a)

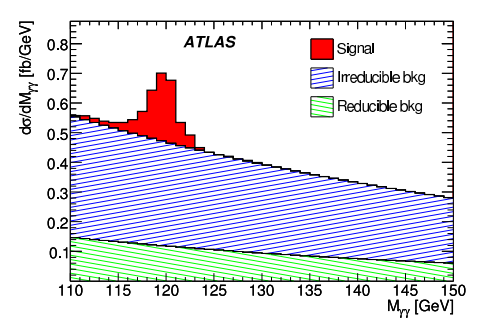

(b)

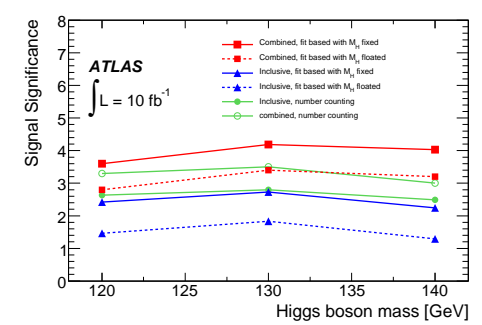

(c)

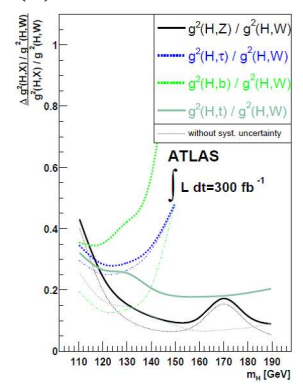

Figure 4: (a) the invariant mass of $\gamma \gamma$ of signal and background with the 2 jets analysis and (b) the expected signal significance of $H \rightarrow \gamma \gamma$ with several methods. (c) uncertainties of measurement of relative couplings as a function of the Higgs mass.

couplings, that is, with respect to the coupling between Higgs and W boson [6]. With $300 \mathrm{fb}^{-1}$ of data, it is possible to measure, e.g. a relative top coupling (squared) with an uncertainty of $15-30 \%$.

\section{Conclusions}

The discovery of a low mass Higgs with both $\tau \tau$ and $\gamma \gamma$ channels at the LHC is promising. The development of background estimations from data is on-going and one of them for VBF $H \rightarrow$ $\tau \tau$ is presented. The existence of low mass Higgs is interesting from a viewpoint of coupling measurements. Top, bottom and tau Yukawa couplings but also couplings to gauge bosons can be determined with a few/several $10 \%$ with $300 \mathrm{fb}^{-1}$ at the LHC.

\section{References}

[1] ATLAS Collaboration, JINST 3 (2008), S08003

[2] CMS Collaboration, JINST 3 (2008), S08004

[3] ATLAS Collaboration, CERN-OPEN-2008-020 [arXiv: 0901 .0512]

[4] CMS Collaboration, [CMS PAS HIG-08-001] and [CMS PAS HIG-08-008]

[5] CMS Collaboration, J.Phys.G34:995-1579 (2007)

[6] M. Dührssen, ATL-PHYS-2003-030 